\title{
Application of Benchtop-magnetic resonance imaging in a nude mouse tumor model
}

Henrike Caysa ${ }^{1,2}$, Hendrik Metz ${ }^{1}$, Karsten Mäder ${ }^{1}$ and Thomas Mueller²

\begin{abstract}
Background: MRI plays a key role in the preclinical development of new drugs, diagnostics and their delivery systems. However, very high installation and running costs of existing superconducting MRI machines limit the spread of MRI. The new method of Benchtop-MRI (BT-MRI) has the potential to overcome this limitation due to much lower installation and almost no running costs. However, due to the low field strength and decreased magnet homogeneity it is questionable, whether BT-MRI can achieve sufficient image quality to provide useful information for preclinical in vivo studies. It was the aim of the current study to explore the potential of BT-MRI on tumor models in mice.

Methods: We used a prototype of an in vivo BT-MRI apparatus to visualise organs and tumors and to analyse tumor progression in nude mouse xenograft models of human testicular germ cell tumor and colon carcinoma.

Results: Subcutaneous xenografts were easily identified as relative hypointense areas in transaxial slices of NMR images. Monitoring of tumor progression evaluated by pixel extension analyses based on NMR images correlated with increasing tumor volume calculated by calliper measurement. Gd-BOPTA contrast agent injection resulted in a better differentiation between parts of the urinary tissues and organs due to fast elimination of the agent via kidneys. In addition, interior structuring of tumors could be observed. A strong contrast enhancement within a tumor was associated with a central necrotic/fibrotic area.
\end{abstract}

Conclusions: BT-MRI provides satisfactory image quality to visualize organs and tumors and to monitor tumor progression and structure in mouse models.

\section{Background}

MRI plays a key role in the preclinical development of new drugs, diagnostics and their delivery systems. However, very high installation and running cost of existing superconducting MRI machines limit the spread of the method. The new method of Benchtop-MRI (BT-MRI) has the potential to overcome this limitation due to much lower installation and almost no running costs. The lower quality of the NMR images is expected due to the low field strength and decreased magnet homogeneity. However, very recently we could show that BTMRI is able to characterize floating mono- or bilayer tablets, osmotic controlled push-pull tablets [1-4] or scaffolds for tissue engineering in vitro [5]. A broad, important and increasing range of MRI applications are

\footnotetext{
* Correspondence: thomas.mueller@medizin.uni-halle.de

${ }^{2}$ Martin-Luther-University Halle-Wittenberg, Department of Internal Medicine IV, Oncology/Hematology, Ernst-Grube-Str. 40, 06120 Halle/Saale, Germany Full list of author information is available at the end of the article
}

linked with preclinical studies on small rodents such as mice or rats [6-8]. Thereby, first developments and testing of more compact MRI systems have been reported $[9,10]$. In the present study we have tested a prototype of a new in vivo BT-MRI apparatus.

Clearly, BT-MRI could overcome one of the current main limitations of preclinical MRI, the high costs. However, the question arises, whether BT-MRI can achieve sufficient image quality to provide useful information for preclinical in vivo studies. In a recent paper we have demonstrated that BT-MRI can be used to characterize in situ forming implants in mice [11]. A major application field of preclinical MRI is linked to cancer research. It was therefore the aim of the current study to explore the potential of BT-MRI on tumor models in mice. Nude mouse xenograft models of different human tumors were used to test the suitability of the new BT-MRI system for visualisation of organs and tumors and for quantification of tumor progression.

\section{Biomed Central}




\section{Methods}

\section{NMR system and its characteristics}

A $21 \mathrm{MHz}$ NMR benchtop prototype system "MARAN DRX2" (Oxford Instruments) capable of imaging with a horizontal bore of $23 \mathrm{~mm}$ diameter was used (Figure $1)$. The instrument is equipped with a temperature control unit and capable of $\mathrm{T}_{1}$ and $\mathrm{T}_{2}$ relaxation measurements, the determination of diffusion coefficients and imaging.

\section{NMR imaging parameter}

The temperature was set to $37^{\circ} \mathrm{C}$. Always 4 slices were simultaneously measured with: slice distance: $3.5 \mathrm{~mm}$, slice width: $3 \mathrm{~mm}$, spin echo time TE: $9.8 \mathrm{~ms}$, repetition time TR: $172 \mathrm{~ms}$, averages: 32 or 16 (for time critical kinetics), total time: 715 s or 357 s, respectively, FOV: 40*40 mm. The pulse sequence was T2SE.

The MRI acquisition parameters were optimized under some hardware restrictions. TE is limited by the bandwidth of $10 \mathrm{KHz}$ to $9.8 \mathrm{~ms}$. An increase of the bandwidth allows shorter TE, however it leads also to stronger image distortions. A TR value of $150 \mathrm{~ms}$ gives an optimal contrast for marbled meat and also for mice. For 4 slices TR is limited to $171.4 \mathrm{~ms}$. Therefore $172 \mathrm{~ms}$ was used for TR as a good compromise between best contrast and simultaneous acquisition of 4 slices. The resulting images are therefore $\mathrm{T} 1$-weighted and range from hyperintense signals for fatty tissues to hypointense signals for water. The higher number of averages was chosen to improve the signal-to-noise ratio. For kinetics of contrast agent distribution a rapid image acquisition may be essential. Therefore measurements with lesser averages were also performed, even though the image quality is reduced.

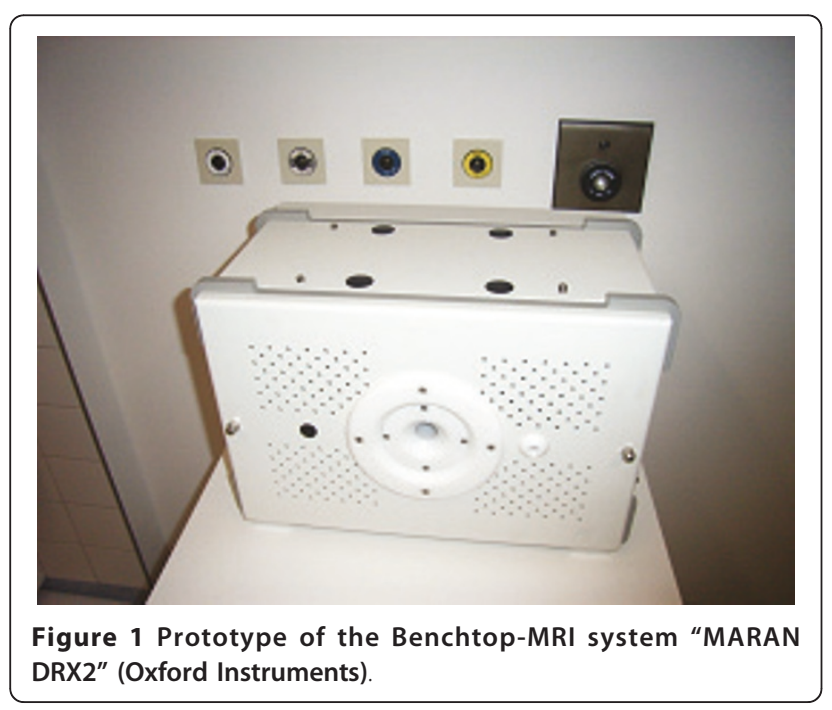

Cell culture, xenograft tumor model, measurements and analyses

Human colon carcinoma cell lines DLD-1, HCT8 and HT29 and human testicular germ cell tumor cell line $1411 \mathrm{HP}$ were maintained as monolayer cultures in RPMI-1640 with 10\% FCS and streptomycin/penicillin. Cultures were grown at $37^{\circ} \mathrm{C}$ in a humidified atmosphere of $5 \% \mathrm{CO}_{2} / 95 \%$ air.

Eight week old male athymic-nude Foxn1 nu/nu mice (Harlan Winkelmann, Germany) were injected s.c. with $3 \times 10^{6}$ tumor cells in both flanks. NMR Imaging of mice was performed once a week. For comparison, the size of the xenograft tumors was also measured by means of a calliper. For imaging with a positive MRI contrast agent mice received $150 \mu \mathrm{l}$ of gadobenate dimeglumine (Gd-BOPTA; $0.03 \mathrm{mmol} / \mathrm{kg}$ in $0.9 \% \mathrm{NaCl}$ ) via tail vein injection. For investigation of contrast agent associated effects with special focus on xenograft tumors the dose of Gd-BOPTA was increased according to dosage applied in men $(0.1 \mathrm{mmol} / \mathrm{kg})$. Animals were anaesthetised via i.p. application of ketamine/xylazine mixture prior to imaging. Body weight was assessed twice weekly. For histological examination tumors were explanted, fixed in $4 \%$ formalin and embedded in paraffin. Hematoxylin/Eosin staining of slices was performed according to standard protocols. All animal protocols were approved by the laboratory animal care and use committee of Sachsen-Anhalt, Germany.

Quantification of xenograft tumor growth was performed by

1.) volume calculation based on calliper measurements using the formula $\mathbf{a}^{2} \times \mathbf{b} \times \mathbf{\pi} / \mathbf{6}$ with $\mathbf{a}$ being the short and $\mathbf{b}$ the long dimension and

2.) measurement of pixel extensions of tumor sections based on NMR images $(128 \times 128$ JPG) using the measure tool of GNU Image Manipulation Program (GIMP 2.6.8) and calculating the area using formula $\mathbf{A}=\mathbf{a} / \mathbf{2} \times$ $\mathbf{b} / 2 \times \pi$.

\section{Results}

Imaging of organs and tumors; gadobenate dimeglumine (Gd-BOPTA) induced MRI contrast

A nude mouse xenograft model of different human tumors was used to determine the image sensitivity and quality of the BT-MRI system. Gd-BOPTA as one of the clinically used low molecular weight gadolinium chelates was selected for contrast agent enhanced MRI. A good differentiation between cortex of kidney and renal pelvis could be observed depending on circulation time of the contrast agent (Figure 2A). Furthermore, the fast renal elimination of Gd-BOPTA was visualised. The urinary bladder was visible as a bright, hypertense sphere unlike the NMR image without contrast agent (Figure 2B). Subcutaneous xenograft tumors 

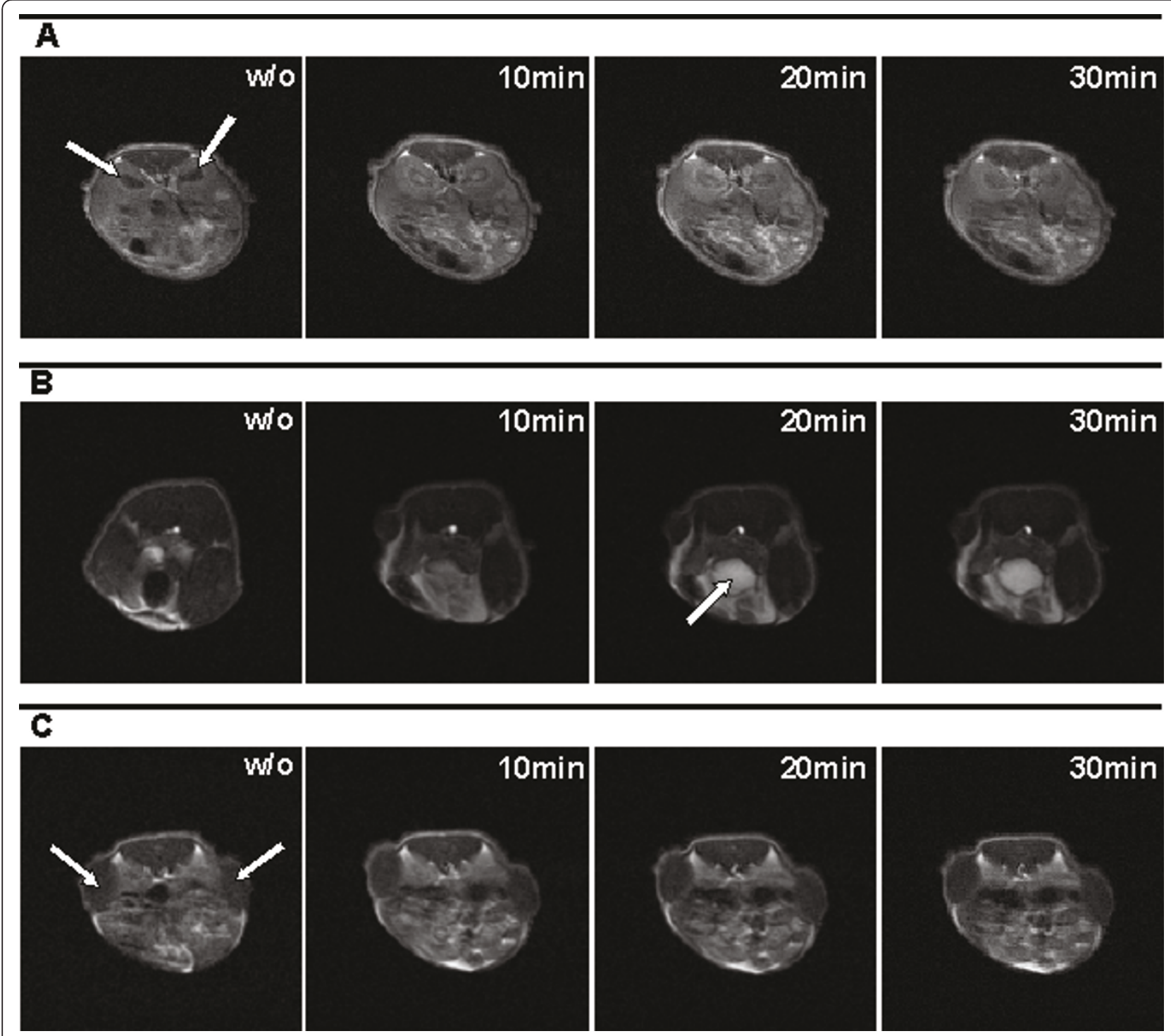

Figure 2 Transaxial NMR images of mice (face-down position) bearing two s.c. xenografts; left: 1411HP germ cell tumor, right: DLD-1 colon carcinoma. Images were taken without Gd-BOPTA and $10 \mathrm{~min}, 20 \mathrm{~min}$ and $30 \mathrm{~min}$ after i.v. application of Gd-BOPTA. (A): The illustration of renal pelvis was clearly enhanced directly after contrast agent injection in light grey compared to a black central area without Gd-BOPTA. The fast nephritic elimination caused a signal decrease (darker grey) already after $30 \mathrm{~min}$. White arrows point at kidneys. (B): High contrast enhancement in the urinary bladder (white arrow) was identifiable as hypertense area compared to a hypotense one without contrast agent. (C): Subcutaneous xenograft tumors are visible as relative hypointense area (white arrows).

were easily identified as relative hypointense area at each body site (Figure 2C).

To study the contrast agent associated effects with special focus on xenograft tumors we used a higher dose of Gd-BOPTA according to dosage applied in men. As shown in Figure 3A an interior structuring of tumors could be observed. This was characterized by time dependent alterations of contrast enhancement with initial enhancement of the tumor rim followed by a centripetal progression of the signal. In one case of a strong central contrast enhancement (Figure 3B) the tumor was explanted, fixed and slices were analysed histologically after HE staining. A large central necrotic/fibrotic area could be observed surrounded by peripherally arranged vital tumor cells (Figure 3C).

\section{Monitoring of xenograft tumor growth}

Apart from tumor detection the quantification of tumor burden is one important aspect of non-invasive in vivo imaging techniques. To test whether the BT- 


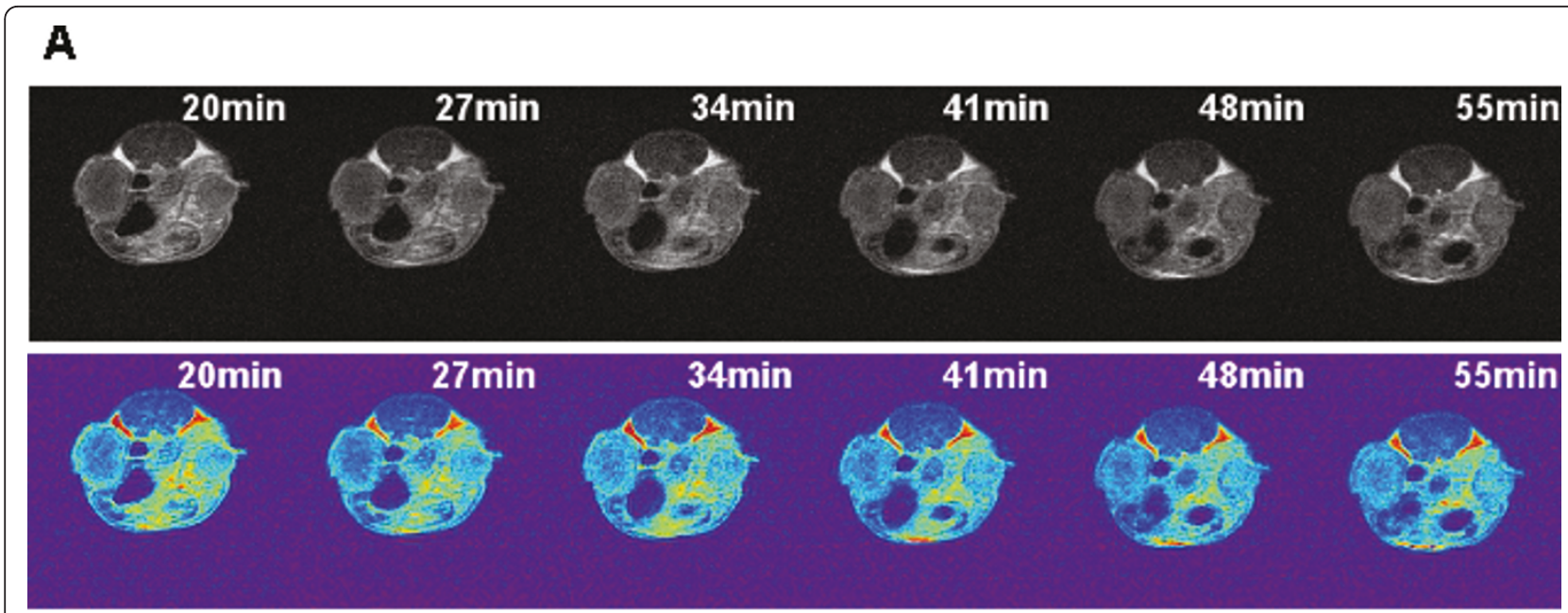

$\mathbf{B}$
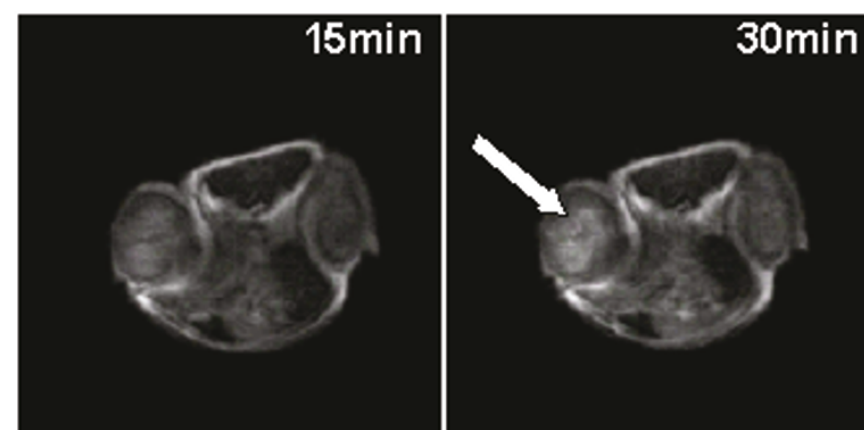

C

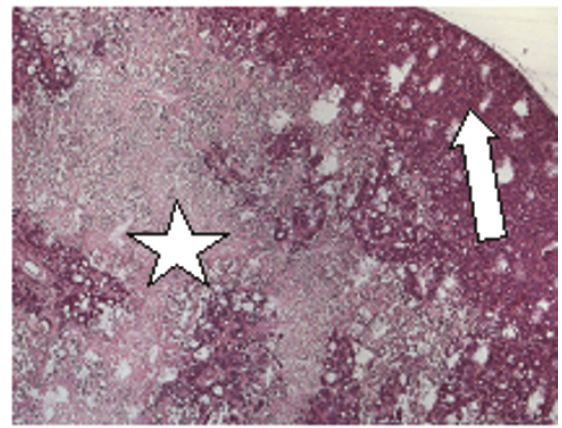

Figure 3 Analysis of contrast agent induced interior structuring of tumours. (A): Transaxial NMR images of a mouse (face-down position) bearing two s.c. xenografts; left: HT29 colon carcinoma, right HCT8 colon carcinoma. Images were taken to the indicated time points after i.V. application of higher dosed Gd-BOPTA $(0.1 \mathrm{mmol} / \mathrm{kg})$. A time dependent alteration of contrast enhancement with initial enhancement of the tumor rim followed by a centripetal progression of the signal is observed in the HT29 tumor. The HCT8 tumor was too small for detailed analyses although a time dependent alteration of the signal could also be observed. (upper panel - grayscale, lower panel - pseudocolor) (B): Transaxial NMR images of a mouse (face-down position) bearing two s.c. HT29 xenografts 15 min and 30 min after i.v. application of Gd-BOPTA. One tumor showed strong contrast enhancement and an interior structuring could be observed (white arrow). (C): HE staining of the well structured left HT29 xenograft shown in (A). Depicted is a section at the side of the tumor to represent the whole structure composed of a large central necrotic/fibrotic area (white star) surrounded by peripherally arranged vital tumor cells (white arrow).

MRI system is suitable for following s.c. xenograft growth the tumor burden was examined in 2 groups of 3 mice each bearing 2 different tumors: one group with 1411 HP germ cell tumor and DLD-1 colon carcinoma, one group with HT29 colon carcinoma and DLD-1 colon carcinoma. Growth of tumors was followed using (a) calliper measurement and volume calculation and (b) BT-MRI and measurement of pixel extensions of tumor sections based on NMR images. For both methods comparable progression profiles could be observed, which was independent of GdBOPTA injection. A representative example of one individual is presented in Figure 4A and 4B. In addition, all values calculated by pixel extension analyses were plotted dependent on respective values calculated by calliper measurement. This demonstrates the correlation of both applications (Figure 4C).

\section{Discussion}

MRI as a non-invasive imaging technology plays a key role in preclinical in vivo evaluation of tumor therapies. The development of a BT-MRI system for small animal imaging could lead to easy detection of tumor mass and progression with little effort and low costs. Additionally, MRI provides an insight into organs and tissues of laboratory animals.

The experimental results clearly proof that BT-MRI can be used to visualise organs and tumors in nude mouse xenograft models. Subcutaneous xenografts were easily identified as relative hypointense areas in transaxial slices of NMR images. In addition BT-MRI system is suitable for following xenograft tumor growth. Monitoring of tumor progression evaluated by pixel extension analyses based on NMR images correlated with increasing tumor volume calculated by 


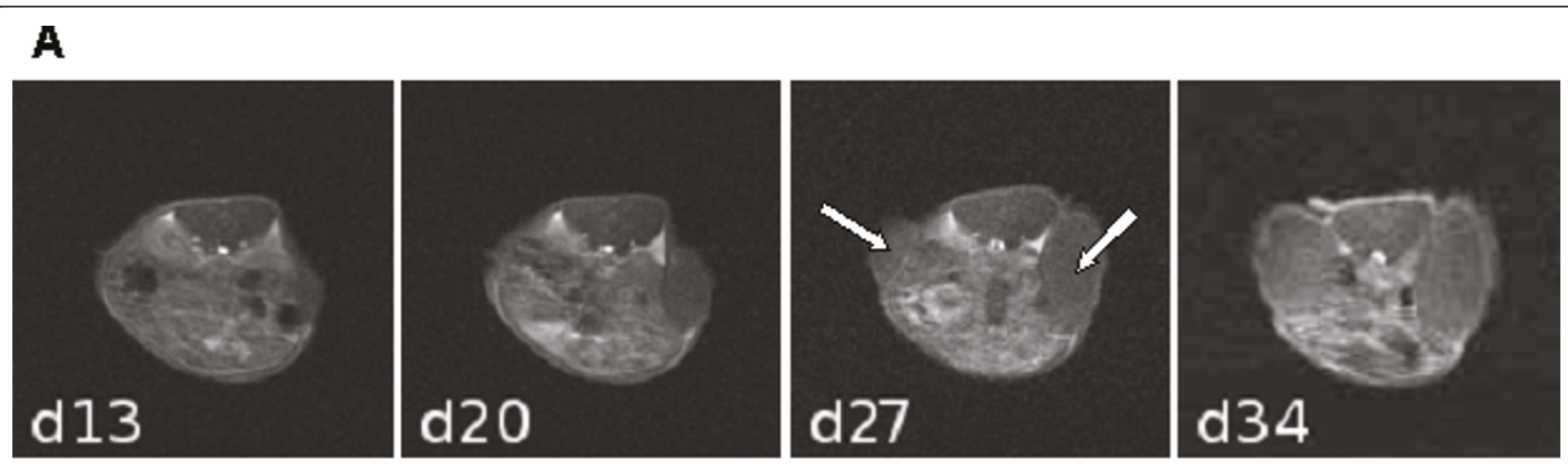

B

$1411 \mathrm{HP}$ xenograft (left flank)
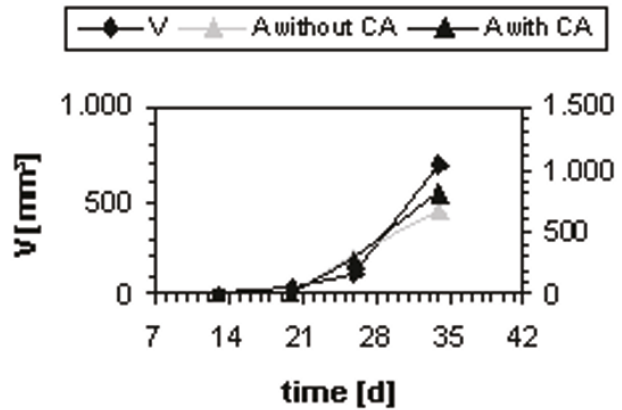

C

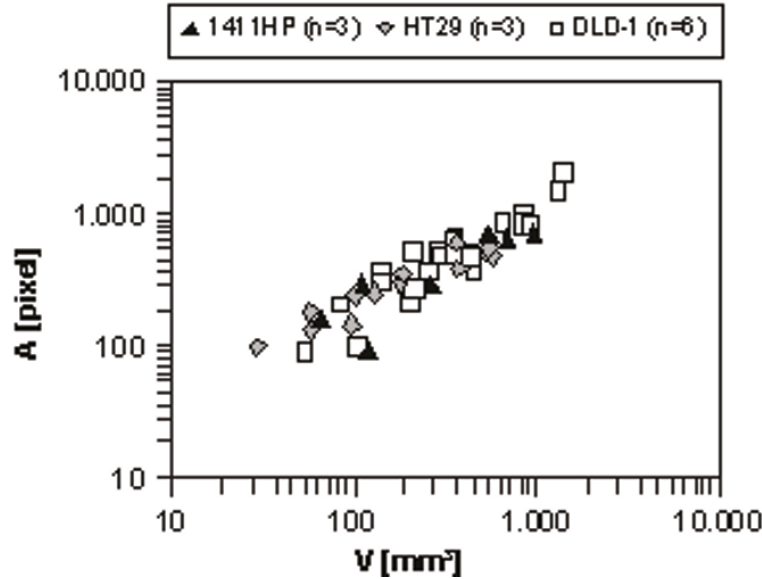

\section{DLD-1 xenograft (right flank)}
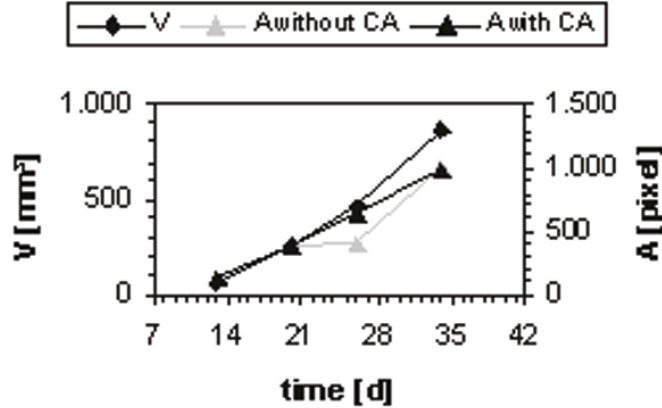

Figure 4 Monitoring of xenograft tumor growth. (A): Transaxial NMR images of a mouse (face-down position) bearing two s.c. xenografts (left: $1411 \mathrm{HP}$ germ cell tumor, right: DLD-1 colon carcinoma) analysed over 5 weeks (d13, d20, d27, d34 post cell injection). Depicted images were taken 10 min after i.v. application of Gd-BOPTA. White arrows point at tumors. (B): Following tumor growth of example shown in Figure 4A as analysed by calliper measurements and volume calculation compared to analyses by pixel extension of tumor sections based on NMR images (with or without GdBOPTA (CA)). Both tumor volume ( $V$ ) and tumor section extent (A) comparably increased over the observation period. (C): Correlation of both methods: calculation of tumor growth by calliper measurement $(V)$ and pixel extension analyses based on NMR images (A) of all 12 tumors.

calliper measurement. This is an important requirement for application of BT-MRI system in orthotopic/ metastatic tumor models to evaluate the whole tumor burden. For this purpose it is necessary to take serial slices of NMR images to get the largest dimension of the tumor as basis for calculation. In addition the whole tumor shape can be reconstituted.

One critical aspect using orthotopic/metastatic tumor models could be the visualization of metastasis in tissues and organs depending on the model. This may require 
application of contrast agent for differentiation between tumor and normal tissue. In this study we used GdBOPTA as one of the clinically used low molecular weight gadolinium chelates. Gd chelates are commonly used as MRI contrast agents for the detection of solid tumors in patients where an initial tumor rim enhancement is usually observed [12-18]. Thereby the characteristic enhancement of the tumor rim can be used for the differentiation between malignant and benign masses [15]. Initially most tumors in our study showed no peripheral contrast enhancement on NMR images. Applying a higher but well tolerated dose of Gd-BOPTA such an effect could be observed, albeit not in each case. This may be due to the artificial location of the tumor as subcutaneous xenograft. Moreover, it was observed that low molar mass Gd chelates show an initial rim enhancement, followed by a washout effect, which requires that the images are obtained within the first 2 min after injection [19]. This probably explains the lack of initial rim enhancement in our models after application of low dose Gd-BOPTA. In this regard the application of macromolecular MRI contrast agents could be useful [20]. They have a longer circulation time and are more confined to the blood pool, therefore giving a longer time window for imaging in mice models.

A main advantage of MRI is the capability to characterize important tumor characteristics (e.g. internal structure, oedema in the tumor environment, necrotic areas). We observed a pronounced interior structuring of an s.c. HT29 tumor after i.v. injection of the contrast agent Gd-BOPTA. Histological analyses revealed that a large central necrotic/fibrotic area was associated with contrast enhancement. Such an effect can also be observed in patient tumors. After the characteristic initial tumor rim enhancement a centripetal progression of the signal can occur depending on the tumor structure, e.g. determined by different vascular architecture $[12,15,21]$. Early peripheral enhancement with centripetal progression was seen in invasive carcinomas with a high peripheral and a low central microvessel density, which was associated with fibrosis and/or necrosis $[12,21]$. This demonstrates that depending on the tumor and used contrast agent the BT-MRI system is suitable for observation of intratumoral structures and that characteristic features of patient tumors can be reproduced in the model system. It offers the opportunity to follow intratumoral processes under therapy.

Further work will be done particularly with regard to imaging of different orthotopic installed tumors and their progression as well as the development of metastatic disease. Other contrast agents will also be examined in order to find better enhancement of (small) tumor sites and metastases. Moreover, other contrast enhancer could lead to better results for imaging of interior tumor structures.

\section{Conclusions}

The results of the current study show that BT-MRI is, despite its limitations with respect to the magnetic field strength and magnet homogeneity, clearly capable of providing satisfactory image slice quality to visualize organs and tumors and to monitor tumor progression in mouse models.

\section{List of abbreviations}

MRI: magnetic resonance imaging; BT-MRI: benchtop-magnetic resonance imaging; NMR: nuclear magnetic resonance; Gd-BOPTA: gadobenate dimeglumine; s.c.: subcutaneous; HE: hematoxylin/eosin

\section{Acknowledgements}

We would like to thank Dr. lan Nicholson and his colleagues from Oxford Instruments for the development, manufacture and installation of the BT-MRI prototype apparatus.

The study was supported in part by grants from the Federal State of Saxonia-Anhalt (FKZ 3646A/0907).

\section{Author details}

${ }^{1}$ Martin-Luther-University Halle-Wittenberg, Department of Pharmaceutics and Biopharmaceutics, Wolfgang-Langenbeck-Str. 4, 06114 Halle/Saale, Germany. ${ }^{2}$ Martin-Luther-University Halle-Wittenberg, Department of Internal Medicine IV, Oncology/Hematology, Ernst-Grube-Str. 40, 06120 Halle/Saale, Germany.

\section{Authors' contributions}

$\mathrm{HC}, \mathrm{HM}, \mathrm{KM}$ and TM designed the study. HC, HM and TM performed experiments. HC, HM, KM and TM analysed data. $\mathrm{HC}$ and TM wrote the paper. All gave final approval.

\section{Competing interests}

The authors declare that they have no competing interests.

Received: 21 February 2011 Accepted: 21 July 2011

Published: 21 July 2011

\section{References}

1. Malaterre V, Metz H, Ogorka J, Gurny R, Loggia N, Mader K: Benchtopmagnetic resonance imaging (BT-MRI) characterization of push-pull osmotic controlled release systems. J Control Release 2009, 133:31-36.

2. Metz H, Mader K: Benchtop-NMR and MRI - a new analytical tool in drug delivery research. Int J Pharm 2008, 364:170-175.

3. Strubing S, Abboud T, Contri RV, Metz H, Mader K: New insights on poly (vinyl acetate)-based coated floating tablets: characterisation of hydration and $\mathrm{CO} 2$ generation by benchtop MRI and its relation to drug release and floating strength. Eur J Pharm Biopharm 2008, 69:708-717.

4. Strubing S, Metz H, Mader K: Characterization of poly(vinyl acetate) based floating matrix tablets. J Control Release 2008, 126:149-155.

5. Nitzsche H, Metz H, Lochmann A, Bernstein A, Hause G, Groth T, Mader K: Characterization of scaffolds for tissue engineering by benchtopmagnetic resonance imaging. Tissue Eng Part C Methods 2009, 15:513-521.

6. Benoit MR, Mayer D, Barak Y, Chen IY, Hu W, Cheng Z, Wang SX, Spielman DM, Gambhir SS, Matin A: Visualizing implanted tumors in mice with magnetic resonance imaging using magnetotactic bacteria. Clin Cancer Res 2009, 15:5170-5177.

7. McConville P, Hambardzumyan D, Moody JB, Leopold WR, Kreger AR, Woolliscroft MJ, Rehemtulla A, Ross BD, Holland EC: Magnetic resonance imaging determination of tumor grade and early response to temozolomide in a genetically engineered mouse model of glioma. Clin Cancer Res 2007, 13:2897-2904. 
8. Brockmann MA, Kemmling A, Groden C: Current issues and perspectives in small rodent magnetic resonance imaging using clinical MRI scanners. Methods 2007, 43:79-87.

9. Inoue $Y$, Nomura Y, Haishi T, Yoshikawa K, Seki T, Tsukiyama-Kohara K, Kai C, Okubo T, Ohtomo K: Imaging living mice using a 1-T compact MRI system. J Magn Reson Imaging 2006, 24:901-907.

10. Shirai T, Haishi T, Utsuzawa S, Matsuda Y, Kose K: Development of a compact mouse MRI using a yokeless permanent magnet. Magn Reson Med Sci 2005, 4:137-143.

11. Kempe $S$, Metz $H$, Pereira $P G$, Mader $K$ : Non-invasive in vivo evaluation of in situ forming PLGA implants by benchtop magnetic resonance imaging (BT-MRI) and EPR spectroscopy. Eur J Pharm Biopharm 2009.

12. Buadu LD, Murakami J, Murayama S, Hashiguchi N, Sakai S, Toyoshima S, Masuda K, Kuroki S, Ohno S: Patterns of peripheral enhancement in breast masses: correlation of findings on contrast medium enhanced MRI with histologic features and tumor angiogenesis. J Comput Assist Tomogr 1997, 21:421-430.

13. Geirnaerdt MJ, Bloem JL, van der Woude HJ, Taminiau AH, Nooy MA, Hogendoorn PC: Chondroblastic osteosarcoma: characterisation by gadolinium-enhanced MR imaging correlated with histopathology. Skeletal Radiol 1998, 27:145-153.

14. Kuhl CK: MRI of breast tumors. Eur Radiol 2000, 10:46-58.

15. Ma LD, Frassica FJ, McCarthy EF, Bluemke DA, Zerhouni EA: Benign and malignant musculoskeletal masses: MR imaging differentiation with rimto-center differential enhancement ratios. Radiology 1997, 202:739-744.

16. Mitchell DG, Saini S, Weinreb J, De Lange EE, Runge VM, Kuhlman JE, Parisky Y, Johnson CD, Brown JJ, Schnall M, et al: Hepatic metastases and cavernous hemangiomas: distinction with standard- and triple-dose gadoteridol-enhanced MR imaging. Radiology 1994, 193:49-57.

17. Mussurakis S, Gibbs P, Horsman A: Peripheral enhancement and spatial contrast uptake heterogeneity of primary breast tumors: quantitative assessment with dynamic MRI. J Comput Assist Tomogr 1998, 22:35-46.

18. Tsien C, Gomez-Hassan D, Ten Haken RK, Tatro D, Junck L, Chenevert TL, Lawrence T: Evaluating changes in tumor volume using magnetic resonance imaging during the course of radiotherapy treatment of high-grade gliomas: Implications for conformal dose-escalation studies. Int J Radiat Oncol Biol Phys 2005, 62:328-332.

19. Morris EA: Breast cancer imaging with MRI. Radiol Clin North Am 2002 40:443-466.

20. Daldrup H, Shames DM, Wendland M, Okuhata Y, Link TM, Rosenau W, Lu Y, Brasch RC: Correlation of dynamic contrast-enhanced MR imaging with histologic tumor grade: comparison of macromolecular and smallmolecular contrast media. AJR Am J Roentgenol 1998, 171:941-949.

21. Buadu LD, Murakami J, Murayama S, Hashiguchi N, Sakai S, Masuda K, Toyoshima S, Kuroki S, Ohno S: Breast lesions: correlation of contrast medium enhancement patterns on MR images with histopathologic findings and tumor angiogenesis. Radiology 1996, 200:639-649.

doi:10.1186/1756-9966-30-69

Cite this article as: Caysa et al: Application of Benchtop-magnetic resonance imaging in a nude mouse tumor model. Journal of Experimental \& Clinical Cancer Research 2011 30:69.

\section{Submit your next manuscript to BioMed Central and take full advantage of:}

- Convenient online submission

- Thorough peer review

- No space constraints or color figure charges

- Immediate publication on acceptance

- Inclusion in PubMed, CAS, Scopus and Google Scholar

- Research which is freely available for redistribution 